\title{
Accuracy of Heart Rate Measurement Using Smartphones During Treadmill Exercise in Male Patients With Ischemic Heart Disease
}

\author{
Eun Sun Lee, $\mathrm{MD}^{1}$, Jin Seok Lee, $\mathrm{PhD}^{2}$, Min Cheol Joo, $\mathrm{MD}^{1}$, Ji Hee Kim, $\mathrm{MD}^{1}$, Se Eung Noh, $\mathrm{MD}^{1}$ \\ ${ }^{1}$ Department of Rehabilitation Medicine and Institute of Wonkwang Medical Science, Wonkwang University \\ School of Medicine, Iksan; ${ }^{2}$ Department of Biomedical Engineering, Wonkwang University School of Medicine, Iksan, Korea
}

\begin{abstract}
Objective To evaluate the accuracy of a smartphone application measuring heart rates (HRs), during an exercise and discussed clinical potential of the smartphone application for cardiac rehabilitation exercise programs.

Methods Patients with heart disease (14 with myocardial infarction, 2 with angina pectoris) were recruited. Exercise protocol was comprised of a resting stage, Bruce stage II, Bruce stage III, and a recovery stage. To measure HR, subjects held smartphone in their hands and put the tip of their index finger on the built-in camera for 1 minute at each exercise stage such as resting stage, Bruce stage II, Bruce stage III, and recovery stage. The smartphones recorded photoplethysmography signal and HR was calculated every heart beat. HR data obtained from the smartphone during the exercise protocol was compared with the HR data obtained from a Holter electrocardiography monitor (control).

Results In each exercise protocol stage (resting stage, Bruce stage II, Bruce stage III, and the recovery stage), the HR averages obtained from a Holter monitor were $76.40 \pm 12.73,113.09 \pm 14.52,115.64 \pm 15.15$, and $81.53 \pm 13.08$ bpm, respectively. The simultaneously measured HR averages obtained from a smartphone were $76.41 \pm 12.82$, $112.38 \pm 15.06,115.83 \pm 15.36$, and $81.53 \pm 13 \mathrm{bpm}$, respectively. The intraclass correlation coefficient (95\% confidence interval) was 1.00 (1.00-1.00), 0.99 (0.98-0.99), 0.94 (0.83-0.98), and 1.00 (0.99-1.00) in resting stage, Bruce stage II, Bruce stage III, and recovery stage, respectively. There was no statistically significant difference between the HRs measured by either device at each stage $(\mathrm{p}>0.05)$.

Conclusion The accuracy of measured HR from a smartphone was almost overlapped with the measurement from the Holter monitor in resting stage and recovery stage. However, we observed that the measurement error increased as the exercise intensity increased.
\end{abstract}

Keywords Heart diseases, Rehabilitation, Heart rate, Smartphone, Exercise

Received June 30, 2016; Accepted September 2, 2016

Corresponding author: Se Eung Noh

Department of Rehabilitation Medicine and Institute of Wonkwang Medical Science, Wonkwang University School of Medicine, 895 Muwang-ro, Iksan 54538, Korea. Tel: +82-63-859-1620, Fax: +82-63-843-1385, E-mail: wonrehab@wonkwang.ac.kr

ORCID: Eun Sun Lee (http://orcid.org/0000-0002-9859-2563); Jin Seok Lee (http://orcid.org/0000-0002-8580-490X); Min Cheol Joo (http://orcid. org/0000-0003-2778-4194); Ji Hee Kim (http://orcid.org/0000-0003-2758-124X); Se Eung Noh (http://orcid.org/0000-0002-8846-3282).

@ This is an open-access article distributed under the terms of the Creative Commons Attribution Non-Commercial License (http://creativecommons.org/ licenses/by-nc/4.0) which permits unrestricted noncommercial use, distribution, and reproduction in any medium, provided the original work is properly cited. Copyright $\odot 2017$ by Korean Academy of Rehabilitation Medicine 


\section{INTRODUCTION}

According to the World Health Organization, 170,000 people die from heart disease annually [1]. In Korea, heart disease has been on the rise for years and now has the second highest mortality rate [2]. In the American Heart Association and American College of Cardiology Foundation guidelines, cardiac rehabilitation programs are a class 1 level recommendation for patients with heart disease because they reduce the recurrence and mortality of heart disease. [3]. Nevertheless, the rate of participation in cardiac rehabilitation exercise programs is still low $[4,5]$. To increase the participation rate, homeand community-based cardiac rehabilitation programs incorporating information and communication technology (ICT) have been proposed [6-10].

Maintaining exercise intensity and taking tolerance tests are important aspects of home- and communitybased cardiac rehabilitation exercise programs that can affect the impact and safety of the programs [11]. Among a number of methods prescribed, targeted heart rate (HR) is often used to determine exercise intensity. To conduct a cardiac rehabilitation exercise program, monitoring HR information during exercise is required. However, most medical practitioners currently instruct patients to measure their HR manually placing a finger on the radial artery and counting the number of pulses while checking the time on their watch. Therefore, it is actually difficult for patients to measure their HR accurately. Fatal and non-fatal adverse events, including cardiac arrest, can occur from inappropriate exercise (i.e., too strenuous or too relaxed exercise). To prevent the adverse events, a patient needs to keep one's HR within a targeted or prescribed HR range. Thus, there is emerging needs for the device to accurately and conveniently monitor the HR of a patient helping guide their exercise intensity based on their measured HR during exercise.

Biomedical engineering technology that records and analyzes skin biosignals with a digital camera has recently been developed. This technology has made it possible to measure respiration rate, identify arrhythmias, measure HR, and analyze HR variability [12-15]. Smartphone applications measuring and monitoring HR (with or without chest-attached type, watch type, and earphone type devices), the number of steps, distance traveled, total exercise time, and calories burned during sports activities are currently available commercially. Several studies evaluating the accuracy of HR measurements, in healthy people using these devices during exercise, have suggested that there is no significant difference in the accuracy of HR measurements, compared with those from electrocardiograms (ECG) $[16,17]$. However, since there have been no studies evaluating these findings in patients with heart disease, it is difficult to apply these results to cardiac rehabilitation exercise programs.

Wearable devices can easily measure HR during exercise, compared with smartphones. However, wearable devices may not be as accessible to patients and may lead to additional financial burdens regarding purchase and maintenance. In addition, devices worn with a chest band can cause discomfort as the chest expands with deep breathing. ECGs also have limitations of frequent electrode replacement and high cost. Electrodes can also lead to skin irritation, and patients' sweat and muscle contractions may create signal interference with the ECG. Because of these limitations, it is difficult to use these devices in rehabilitation programs. Taking all limitations into consideration, measuring HR using smartphones is currently the most economical and accessible tool for patients.

In this study, we investigated the accuracy of HR measurements during exercise using a smartphone and its applications in patients with heart disease. This study was conducted as a preliminary study for the development of smart devices to support cardiac rehabilitation and applications for home- and community-based cardiac rehabilitation exercise programs.

\section{MATERIALS AND METHODS}

\section{Subjects}

The study included 16 subjects. The inclusion criteria were patients with heart disease who could perform treadmill exercises, time since disease onset was 3 months or longer, and their disease was stable (myocardial infarction and angina) (Table 1). Treadmill exercise tolerance tests were conducted before subject selection. Subjects who could perform Bruce protocol stage II and III safety exercises were selected. Subjects with musculoskeletal, medical, and psychological problems that could interfere with the treadmill exercises, and subjects who could not perform Bruce protocol stage II and III safety 
Table 1. Characteristics and diagnosis of subjects

\begin{tabular}{ccllll}
\hline $\begin{array}{c}\text { Subject } \\
\text { no. }\end{array}$ & Age/sex & \multicolumn{1}{c}{ PHx } & \multicolumn{1}{c}{ Dx } & PCI & \multicolumn{1}{c}{ Medication } \\
\hline 1 & $70 / M$ & HTN, hyperlipidemia & VAP & No & CCB \\
2 & $65 / M$ & HTN & STEMI & Yes & CCB, atypical $\alpha, \beta$-blocker \\
\hline 3 & $54 / M$ & HTN, hyperlipidemia & STEMI & Yes & CCB, atypical $\alpha, \beta$-blocker \\
4 & $67 / M$ & HTN, hyperlipidemia & UAP & Yes & Atypical $\alpha, \beta$-blocker \\
5 & $67 / M$ & HTN & STEMI & Yes & $\beta$-blocker, nitrate \\
6 & $44 / M$ & HTN & STEMI & Yes & Amiodarone, atypical $\alpha, \beta$-blocker \\
7 & $47 / M$ & HTN, hyperlipidemia & STEMI & Yes & Atypical $\alpha, \beta$-blocker \\
8 & $67 / M$ & HTN & STEMI & Yes & $\beta$-blocker \\
\hline 9 & $59 / M$ & HTN & STEMI & Yes & $\beta$-blocker \\
10 & $47 / M$ & HTN & NSTEMI & Yes & Atypical $\alpha, \beta$-blocker \\
11 & $50 / M$ & DM & STEMI & Yes & $\beta$-blocker \\
12 & $54 / M$ & None & UAP & Yes & CCB \\
\hline 13 & $50 / M$ & HTN & STEMI & No & Atypical $\alpha, \beta$-blocker \\
14 & $59 / M$ & HTN & STEMI & Yes & Ramipril, torsemide, atypical $\alpha, \beta$-blocker \\
15 & $43 / M$ & HTN, hyperlipidemia & NSTEMI & Yes & Ramipril, $\beta$-blocker \\
\hline 16 & $52 / M$ & HTN & & \\
\hline
\end{tabular}

PHx, past history; Dx, diagnosis; PCI, percutaneous coronary intervention; HTN, hypertension; VAP, variant angina pectoris; UAP, unstable angina pectoris; STEMI, ST elevation myocardial infarction; NSTEMI, non-ST elevation myocardial infarction; CCB, calcium channel blocker; DM, diabetes mellitus.

Table 2. Cardiopulmonary treadmill exercise test of subjects

\begin{tabular}{cccccccc}
\hline $\begin{array}{c}\text { Subject } \\
\text { no. }\end{array}$ & MET $_{\text {max }}$ & $\begin{array}{c}\mathbf{B P}_{\text {rest }} \\
(\mathbf{m m H g})\end{array}$ & $\begin{array}{c}\mathbf{B P}_{\text {max }} \\
(\mathbf{m m H})\end{array}$ & $\begin{array}{c}\mathbf{H R}_{\text {rest }} \\
(\mathbf{b p m})\end{array}$ & $\begin{array}{c}\mathbf{H R}_{\max } \\
(\mathbf{b p m})\end{array}$ & $\begin{array}{c}\mathbf{V O}_{\text {max }} \\
(\mathbf{m L} / \mathbf{k g} / \mathbf{m i n})\end{array}$ & RER \\
\hline 1 & 12.8 & $133 / 75$ & $196 / 46$ & 80 & 140 & & \\
2 & 12.8 & $124 / 76$ & $217 / 80$ & 76 & 142 & 27.4 & 1.04 \\
\hline 3 & 13.4 & $106 / 73$ & $160 / 89$ & 68 & 169 & & \\
4 & 10.1 & $102 / 69$ & $178 / 72$ & 86 & 125 & 20.9 & 1.06 \\
\hline 5 & 12.8 & $88 / 59$ & $183 / 67$ & 70 & 135 & 26.5 & 1.10 \\
\hline 6 & 12.8 & $104 / 70$ & $164 / 98$ & 65 & 153 & 23.2 & 1.21 \\
\hline 7 & 10.1 & $122 / 72$ & $195 / 85$ & 90 & 171 & 26.3 & 1.35 \\
\hline 8 & 12.8 & $120 / 70$ & $201 / 90$ & 71 & 126 & 27.7 & 1.10 \\
\hline 9 & 12.8 & $90 / 76$ & $168 / 79$ & 86 & 120 & 15.5 & 1.14 \\
\hline 10 & 12.8 & $133 / 67$ & $191 / 63$ & 93 & 165 & 28.4 & 1.10 \\
\hline 11 & 10.1 & $106 / 74$ & $220 / 88$ & 65 & 138 & 24.1 & 1.22 \\
\hline 12 & 13.8 & $101 / 76$ & $193 / 82$ & 55 & 134 & & 1.19 \\
\hline 13 & 12.8 & $126 / 83$ & $206 / 65$ & 77 & 149 & 17.9 & 1.19 \\
\hline 14 & 14.8 & $124 / 73$ & $161 / 77$ & 52 & 152 & 32 & 1.14 \\
\hline 15 & 14.8 & $84 / 74$ & $159 / 89$ & 54 & 146 & 25.6 & 1.14 \\
\hline 16 & 12.8 & $120 / 74$ & $147 / 62$ & 67 & 135 & 26.4 & 1.26 \\
\hline
\end{tabular}

$\mathrm{MET}_{\max }$, maximal metabolic equivalent; $\mathrm{BP}$, blood pressure; $\mathrm{HR}$, heart rate; $\mathrm{VO}_{2 \max }$, maximal oxygen consumption (mL/ $\mathrm{kg} / \mathrm{min})$; RER, respiratory exchange ratio. 
exercises were excluded from the study (Table 2).

\section{Methods}

Subjects who participated in the cardiac rehabilitation exercise program after visiting the Department of Cardiology in Wonkwang University Hospital (WKUH) were recruited. We ensured subjects' safety by conducting the clinical trial in the cardiac rehabilitation room in the hospital, which was equipped with devices for emergency measures and was under the supervision of medical staff. In addition, this study was conducted after acquiring patient consent in compliance with the clinical trial ethics of the Institutional Review Board of Wonkwang University Hospital (IRB Clinical Trial No. 201401-CTDV-001).

The Bruce protocol was used to adjust the intensity of the treadmill exercises. We measured HRs in the all stages including the resting stage before exercise, the Bruce stages II and III during the treadmill exercise, and the recovery stage (3-minute rest after completion of exercise). For the HR measurement evaluation, our developed smartphone (iPhone 4S, Apple, Cupertino, CA, USA) application based HRs were compared with the HRs measured from a Holter monitor (GE Healthcare, Milwaukee, WI, USA) (Fig. 1).

The accuracy of HR measurements obtained from a smartphone built-in camera was verified using a method that was previously reported [6]. To measure HR, subjects

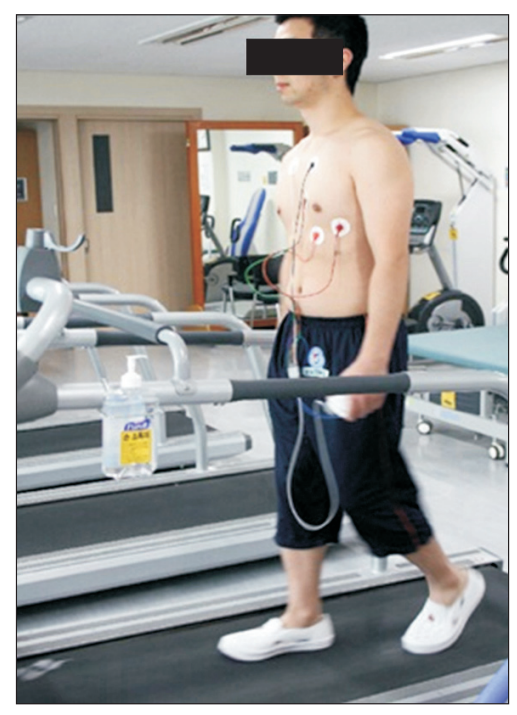

Fig. 1. Subjects' heart rates were measured at rest, during exercise at Bruce stage II, Bruce stage III, and during recovery stage using a smartphone and Holter monitor (GE Healthcare, Milwaukee, WI, USA). held smartphones in their right hands, putting the tip of their right hand's index finger on the camera on the back side of the smartphone, and held it in place with limited movement for 1 minute. We used our previously developed smartphone application, which recorded photoplethysmography (PPG) signals from the index fingertip and calculated HR followed by analyzed heart rate variability, for atrial fibrillation detection [13]. The HR per minute was measured and recorded by the smartphone (Fig. 2). PPG perceives fluctuations in the skin color of patients by monitoring blood flow changes in peripheral blood vessels based on the cardiac activity. It radiates light onto the skin with a light-emitting diode (LED) to increase skin permeability and records images with a camera (Fig. 3). To minimize the noise from patients' motion during exercise, we developed an algorithm to measure the variability in the accuracy of HR measurements. It improved the quality of the signal using two-stage bandpass filtering based on HR. To ensure that HR measurements were as accurate as possible, HR information was also collected with high reliability during exercise using statistical methods, including root mean squared successive difference, Shannon entropy, and turning point ratio during the entire intervals [12-14].

To measure the HR using the Holter monitor (control), electrodes were attached to the chest of patients and ECGs were constantly recorded during all stages of the exercise protocol. HR was then calculated using an exclusive program (MARS, GE Healthcare).

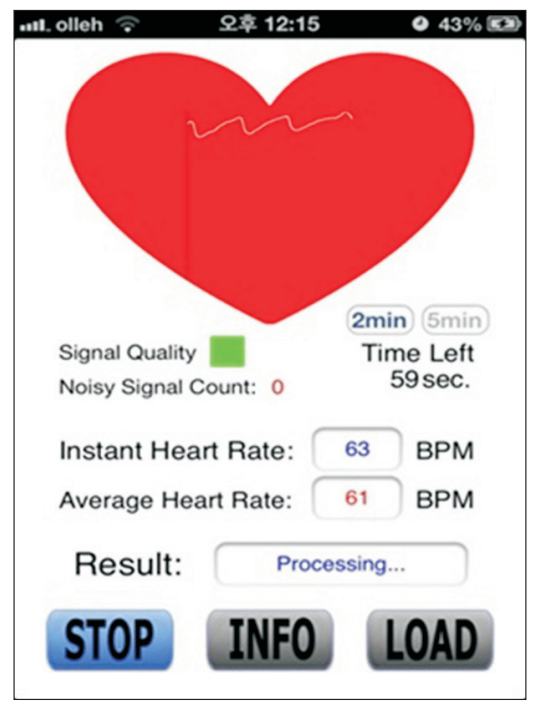

Fig. 2. Atrial fibrillation diagnostic prototype application. 


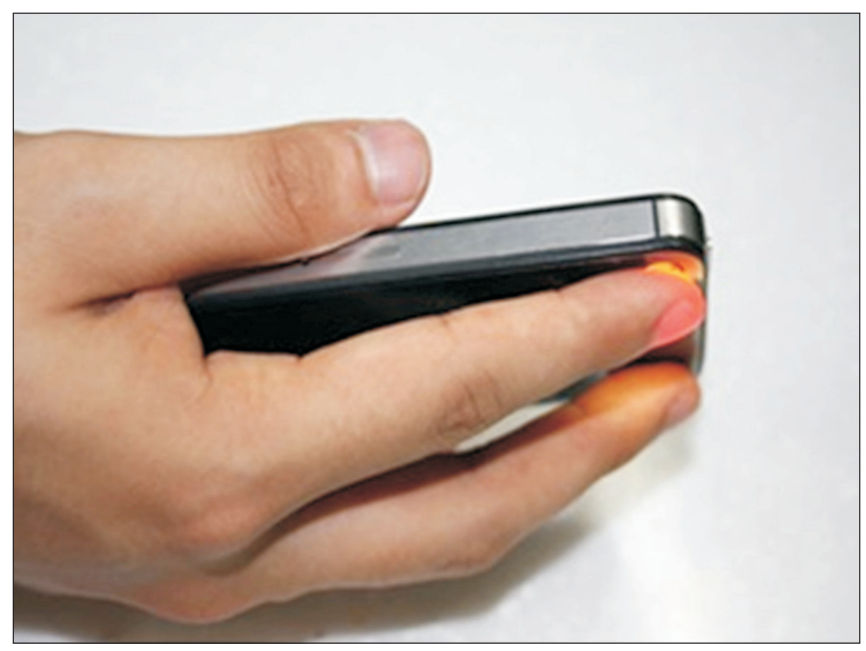

Fig. 3. Subjects held the smartphones in their hands and placed the index finger of their right hands on the camera of the smartphone for 1 minute.

To calculate sample size, we referred to the effect size of a similar pre-existing study [17], setting the effect size $=1.0$, power $(1-\beta$ error probability $)=0.95$, and $\alpha$ error probe $=0.05$. The calculated sample size equaled to 16 using a statistics program (G*Power v3.1.7; Heinrich-Heine University, Dusseldorf, Germany).

To examine the engineered accuracy of HR measurements from our developed smartphone application, we used a Holter monitor as the control to measure HR. In addition, mean absolute error (MAE) and root mean square error (RMSE) of HR measurements using a smartphone were calculated

$$
\begin{aligned}
& M A E=\sum \frac{\left|Y_{\text {iPhone }}-Y_{\text {holter }}\right|}{N}, \\
& R M S E=\sqrt{\sum \frac{\left(Y_{\text {iphone }}-Y_{\text {holter }}\right)^{2}}{N},}
\end{aligned}
$$

where $Y_{\text {iphone }}$ is the HR estimated from the iPhone, and $Y_{\text {holter }}$ is the HR from the Holter monitor. MAE and RMSE are scales that are frequently used to determine the difference of estimated values or values expected by a model, and the ones observed in an actual environment. They are widely used in weather prediction and calculation of engineered accuracy.

\section{Statistical analysis}

SPSS ver. 19.4 (IBM SPSS, Armonk, NY, USA) was used to obtain descriptive statistics of subjects, mean HR, and standard deviation. Paired t-test was also conducted to determine the difference between measuring HR using a Holter monitor versus a smartphone in each stage. The agreement of HR measurements between the two devices was analyzed using the intraclass correlation coefficient. In this study, each subject was assessed by each rater, which was randomly selected (model is 2 ). Reliability was calculated by taking the average of the $k$ rater's measurements (form is $k$ ). Intraclass correlation coefficient was analyzed using a two-way mixed model.

\section{RESULTS}

\section{Patient characteristics}

All sixteen subjects included in the study were men with a mean age of $55.94 \pm 9.05$ years. Eleven subjects had ST elevated myocardial infarction, 3 had non-ST elevated myocardial infarction, and 2 had angina, including variant angina pectoris and unstable angina. In addition, 14 subjects had a history of hypertension, 3 had diabetes, and 5 had hyperlipidemia. Fourteen subjects underwent percutaneous coronary intervention and 2 subjects received conservative medical treatment. Subjects participated in the study while continuing to receive related drug treatment, including calcium channel blockers and $\alpha$ - $\beta$-blockers (Table 1).

\section{Heart rate}

In each exercise stage (resting stage, Bruce stage II, Bruce stage III, and recovery stage), the HR averages obtained from a Holter monitor were $76.40 \pm 12.73,113.09 \pm$ $14.52,115.64 \pm 15.15$, and $81.53 \pm 13.08 \mathrm{bpm}$, respectively. The simultaneously measured HR averages obtained from a smartphone were $76.41 \pm 12.82,112.38 \pm 15.06,115.83 \pm$ 15.36 , and $81.53 \pm 13 \mathrm{bpm}$, respectively.

The average difference (95\% confidence interval) of HR measurements in both devices based on paired t-test results were 0.01 ( -0.10 to 0.13$)$ at rest, $-0.71(-0.85$ to 0.42$)$ during Bruce stage II, 0.18 ( -0.95 to 1.33 ) during Bruce stage III, and 0.10 ( -0.16 to 1.33$)$ during the recovery stage. These differences were not statistically significant ( $p>0.05)$ and had a very small confidence interval ( -0.95 to 1.33) (Table 3).

Coefficients of agreement (95\% confidence interval) for HR measurements between the two devices based on the 
Table 3. Comparison of heart rate between smartphone and Holter monitor

\begin{tabular}{|c|c|c|c|c|}
\hline & \multicolumn{2}{|c|}{ Heart rate (bpm) } & \multirow{2}{*}{$\begin{array}{c}\text { Mean difference } \\
(95 \% \mathrm{CI})\end{array}$} & \multirow{2}{*}{ p-value } \\
\hline & Smartphone & Holter monitor & & \\
\hline Resting & $76.41 \pm 12.8$ & $76.40 \pm 12.73$ & $0.01(-0.10$ to 0.13$)$ & 0.81 \\
\hline Bruce II & $112.38 \pm 15.0$ & $113.09 \pm 14.52$ & $-0.71(-0.85$ to 0.42$)$ & 0.20 \\
\hline Bruce III & $115.83 \pm 15.3$ & $115.64 \pm 15.15$ & $0.18(-0.95$ to 1.33$)$ & 0.73 \\
\hline Recovery & $81.53 \pm 13.0$ & $81.53 \pm 13.08$ & $0.10(-0.16$ to 1.33$)$ & 0.41 \\
\hline
\end{tabular}

Values are presented as mean \pm standard deviation.

CI, confidence interval.

Table 4. Intraclass correlation coefficient $(2, k)$

\begin{tabular}{lcc}
\hline & $\begin{array}{c}\text { ICC } \\
\text { (average measurement) }\end{array}$ & $\mathbf{9 5 \%}$ CI \\
\hline Resting & 1.00 & $1.00-1.00$ \\
Bruce II & 0.99 & $0.98-0.99$ \\
Bruce III & 0.94 & $0.83-0.98$ \\
Recovery & 1.00 & $0.99-1.00$ \\
\hline
\end{tabular}

CI, confidence interval.

intraclass correlation coefficient were $1.00(1.00-1.00)$ in the resting stage, 0.99 (0.98-0.99) in Bruce stage II, 0.94 (0.83-0.98) in Bruce stage III, and 1.00 (0.99-1.00) in the recovery stage. All stages showed high agreement that were statistically significant (Table 4).

\section{Errors in heart rate}

MAEs of HR measured by the smartphone were 0.14 , $0.82,1.10$, and $0.20 \mathrm{bpm}$, compared with HR measured by the Holter monitor in the resting stage, Bruce stage II, Bruce stage III, and the recovery stage, respectively. RMSEs of HR were $0.20,1.84,1.84$, and $0.33 \mathrm{bpm}$ in the resting stage, Bruce stage II, Bruce stage III, and the recovery stage, respectively (Table 5).

\section{DISCUSSION}

Although the participation rate of patients in cardiac rehabilitation exercise programs is low, previous research suggests that the positive effect of cardiac rehabilitation increases in proportion to the number of participants [18]. This highlights the importance of recommending cardiac rehabilitation to as many patients as possible. In 2009, a study by Dunlay et al. [19] evaluating the barriers of participation in cardiac rehabilitation showed that patients' psychological factors, including the perceived
Table 5. Mean absolute error (MAE) and root mean square error (RMSE)

\begin{tabular}{lcc}
\hline & MAE (bpm) & RMSE (bpm) \\
\hline Resting & 0.14 & 0.20 \\
Bruce II & 0.82 & 1.84 \\
Bruce III & 1.10 & 1.84 \\
Recovery & 0.20 & 0.33 \\
\hline
\end{tabular}

necessity and importance of cardiac rehabilitation, had a significant effect on participation. Therefore, it is necessary to identify different approaches and develop auxiliary instruments to enhance the initial participation and continued progress of patients in cardiac rehabilitation. In 2007, data from the Birmingham Rehabilitation Uptake Maximization (BRUM) study found no statistical difference between community-based cardiac rehabilitation programs conducted by a community fitness center and hospital-based cardiac rehabilitation programs [20]. The community-based cardiac rehabilitation program was recommended for patients who could not participate in the hospital-based programs because of geographical and time-based restrictions. However, to implement a similar community-based cardiac rehabilitation program, heart manuals for each individual are needed as well as significant financial resources to pay for, efforts including regular nurse home visits and telephone interviews. Patients must also be able to control their exercise intensity when performing the exercises prescribed by their provider. In 2015, a study evaluating the acceptability of mobile health exercise-based cardiac rehabilitation intervention through text messages found that text messaging was useful in motivating patients with ischemic heart diseases to exercise and continue cardiac rehabilitation programs [6]. What was notable in this study was that the average age of the subjects was 60 years and old- 
er, suggesting that elderly patients may understand and participate in medical intervention programs through mobile devices. Additional intervention programs, smart devices, and systems using information and bioengineering technology are needed to motivate patients to participate in cardiac rehabilitation programs, and continue through them. These developments may also encourage patients to exercise on their own, deeply feel and understand the need for cardiac rehabilitation, and make efforts to conduct the program accurately and safely.

A modified Bruce protocol is usually used to gradually increase exercise intensity in cardiac rehabilitation programs for patients with heart disease. However, in this study the Bruce protocol was used not to perform a clinical exercise test for cardiac rehabilitation treatment, but to observe fluctuations in HR through a quick increase of exercise intensity for a short period of time. Some patients were able to exercise with higher intensity safely. But most of the patients reported difficulties maintaining the hold of the tip of their index finger on the smartphone camera during the treadmill exercise. This was even more difficult when the intensity of the treadmill exercise was increased. It should be noted, that in a preliminary study of healthy volunteers, we observed that the taking the measurements described above were not feasible with Bruce stage IV, which is why we revised the protocol to only include Bruce stage II and Bruce stage III.

When measuring HR during exercise, we assumed that HR measurements using a smartphone were lower than control measurements, because the application algorithm was cancelled in the process of filtering inaccurate signals or that deficits occurred in the PPG input, because fingers were not well attached to the camera because of movement during exercise. In addition, we assumed that HR measurements using a smartphone would be higher than the control HR measurements, because vibrations from exercise were transmitted to the camera via a finger and perceived by the PPG as HR changes, resulting in the addition of signal input. Therefore, to use smart devices in the clinical setting, including cardiac rehabilitation programs, it is essential to develop advanced devices with higher accuracy, that allow the cameras can keep contact with the skin even during higher exercise intensity. Algorithms also need to be improved to better reflect errors from movement during exercise.

Based on the results from this study, we found that measuring the HR of patients with heart disease during all stages of exercise including resting, exercise, and recovery using a smartphone and PPG analytical applications had a high degree of accuracy and may be applicable in the clinical setting, including in cardiac rehabilitation programs.

When comparing the results of this study to recently published intraclass correlation coefficients of HRs measured by smart wrist device ECGs during exercise in healthy volunteers, our results were similar to those obtained with the Apple Watch (0.98, 0.94-0.99), and higher than those obtained with the Fitbit Charge HR (0.78, -0.02-0.93), Samsung Gear S (0.80, 0.40-0.93), and Mio ALPHA (0.91, 0.72-0.97) [16]. In addition, we believe that these results met the criteria for sports activity monitoring applications, including there not being errors of more than 5 HR per minute and there being correlations not less than 0.90 at rest and during activities of moderate intensity [21]. However, it should be noted that limited research exists regarding the accuracy of HR measurements during exercise in patients with heart disease. Unlike healthy subjects, patients with heart disease have several factors, in addition to arrhythmia, that can affect the PPG signal sensor. For example, antihypertensive medicine including calcium channel blockers, $\beta$ blockers, peripheral blood circulation disorders, and hemodynamic fluctuations are assumed to be error factors. As a result, studies evaluating the accuracy of HR measurements with smartphones are needed to determine the feasibility of its clinical application. When interpreting the results of this study, measuring HR with a smartphone was more accurate than measuring HR with wrist band type wearable devices; little difference was observed between HR measurements taken with smartphones and Holter monitors. Subjects can keep their fingertips on the smartphone camera steadily during low-mid intensity treadmill exercise, suggesting that it may be useful in measuring measurements in patients with ischemic heart disease. However, the accuracy of HR measurements deteriorated when exercise intensified. As a result, we believe that technology needs to be developed to better identify errors that may occur during exercises of higher intensity. These developments should also seek to maintain higher accuracy than the current technology, even during exercise, for clinical applications of this technology including cardiac rehabilitation therapy. 
The limitations of this study include the small number of subjects included and the sex distribution of our patient population. Because only a small number of male subjects were recruited, our results cannot be generalized, and several endpoints could not be evaluated. In particular, the effect on heart disease diagnosis could not be evaluated. The effect of underlying diseases such as low blood pressure, autonomic nerve disorder, peripheral vascular diseases, and skin diseases-which are frequently accompanied with heart disease and assumed to affect the measurement of PPG-were also not evaluated. Lastly, HR measurements during Bruce stage IV could not be measured during the treadmill exercise, because it was difficult to keep the fingertip accurately on the camera, as the hand shaking got worse. In fact, although HR monitoring is required during exercise in high-risk patients, all subjects included in the study were low-risk patients. To overcome the limitations described above, further accuracy studies are needed in patients with various diagnoses and heart disease severity including arrhythmia, congestive heart failure, heart valve disease, and patients undergoing cardiac surgery.

We performed a preliminary study to better understand the accuracy and limitations of measuring HR using a smartphone during exercise in patients with heart disease. We realized that better software is needed, including improved algorithms, to reduce measurement errors during exercise. Better hardware is also needed, including auxiliary smart devices for cardiac rehabilitation. Additional clinical trials must be conducted in patients with medical problems combined with various heart diseases.

We believe that in the future, the development, utilization, and application of smart devices to support cardiac rehabilitation will become more prominent. This will also include the development of medical information communication systems, including content to motivate patient participation in cardiac rehabilitation education, as well as patient feedback. The smart devices will also record the intensity and duration of exercise, as well as targeted HR during exercise, to allow patients to better monitor and control their exercise intensity in real time. Lastly, patients will be able to use smart devices to store, transmit, and share information with their provider, who will in turn use the information to make treatment decisions.

With the rise of other biometric technologies [12-15] to monitor patients, autonomic nervous system, blood pressure, blood glucose, respiratory count, estimate of arterial oxygen saturation, as well as arrhythmia diagnoses using HR variability, it is expected that more patients with heart diseases will perform cardiac rehabilitation programs safely and efficiently.

\section{CONFLICT OF INTEREST}

No potential conflict of interest relevant to this article was reported.

\section{ACKNOWLEDGMENTS}

This study was supported from the National Research Foundation of Korea (NRF) funded by the Ministry of Science, ICT \& Future Planning (NRF-2015M3A9D7067215).

\section{REFERENCES}

1. World Health Organization. Prevention of cardiovascular disease: guidelines for assessment and management of cardiovascular risk. Geneva: World Health Organization; 2007.

2. Statistics Korea. Annual report on the cause of death statistics in 2014 [Internet]. Daejeon: Statistics Korea; 2015 [cited 2017 Jan 7]. Available from: http://www. index.go.kr/potal/main/EachDtlPageDetail.do?idx $\mathrm{cd}=1012$.

3. Smith SC Jr, Benjamin EJ, Bonow RO, Braun LT, Creager MA, Franklin BA, et al. AHA/ACCF secondary prevention and risk reduction therapy for patients with coronary and other atherosclerotic vascular disease: 2011 update: a guideline from the American Heart Association and American College of Cardiology Foundation. Circulation 2011;124:2458-73.

4. Witt BJ, Jacobsen SJ, Weston SA, Killian JM, Meverden RA, Allison TG, et al. Cardiac rehabilitation after myocardial infarction in the community. J Am Coll Cardiol 2004;44:988-96.

5. Suaya JA, Shepard DS, Normand SL, Ades PA, Prottas J, Stason WB. Use of cardiac rehabilitation by Medicare beneficiaries after myocardial infarction or coronary bypass surgery. Circulation 2007;116:1653-62.

6. Pfaeffli Dale L, Whittaker R, Dixon R, Stewart R, Jiang Y, Carter K, et al. Acceptability of a mobile health 
exercise-based cardiac rehabilitation intervention: a randomized trial. J Cardiopulm Rehabil Prev 2015;35: 312-9.

7. Leijdekkers P, Gay V. Personal heart monitoring and rehabilitation system using smart phones. Proceedings of 2006 International Conference on Mobile Business; 2006 Jun 26-27; Copenhagen, Denmark. p. 29.

8. Worringham C, Rojek A, Stewart I. Development and feasibility of a smartphone, ECG and GPS based system for remotely monitoring exercise in cardiac rehabilitation. PLoS One 2011;6:e14669.

9. Strisland F, Svagard I, Seeberg TM, Mathisen BM, Vedum J, Austad HO, et al. ESUMS: a mobile system for continuous home monitoring of rehabilitation patients. Conf Proc IEEE Eng Med Biol Soc 2013;2013: 4670-3.

10. Lee YH, Hur SH, Sohn J, Lee HM, Park NH, Cho YK, et al. Impact of home-based exercise training with wireless monitoring on patients with acute coronary syndrome undergoing percutaneous coronary intervention. J Korean Med Sci 2013;28:564-8.

11. American College of Sports Medicine. ACSM's guidelines for exercise testing and prescription. 9th ed. Philadelphia: Lippincott Williams \& Wilkins; 2013.

12. Scully CG, Lee J, Meyer J, Gorbach AM, GranquistFraser D, Mendelson Y, et al. Physiological parameter monitoring from optical recordings with a mobile phone. IEEE Trans Biomed Eng 2012;59:303-6.

13. Lee J, Reyes BA, McManus DD, Maitas O, Chon KH. Atrial fibrillation detection using an iPhone 4S. IEEE Trans Biomed Eng 2013;60:203-6.
14. Lee J, Chon KH. Time-varying autoregressive modelbased multiple modes particle filtering algorithm for respiratory rate extraction from pulse oximeter. IEEE Trans Biomed Eng 2011;58:790-4.

15. Lee J, Chon KH. An autoregressive model-based particle filtering algorithms for extraction of respiratory rates as high as 90 breaths per minute from pulse oximeter. IEEE Trans Biomed Eng 2010;57:2158-67.

16. Wallen MP, Gomersall SR, Keating SE, Wisløff U, Coombes JS. Accuracy of Heart Rate Watches: Implications for Weight Management. PLoS One 2016;11: e0154420.

17. Crouter SE, Albright C, Bassett DR Jr. Accuracy of polar S410 heart rate monitor to estimate energy cost of exercise. Med Sci Sports Exerc 2004;36:1433-9.

18. Kwan G, Balady GJ. Cardiac rehabilitation 2012: advancing the field through emerging science. Circulation 2012;125:e369-73.

19. Dunlay SM, Witt BJ, Allison TG, Hayes SN, Weston SA, Koepsell E, et al. Barriers to participation in cardiac rehabilitation. Am Heart J 2009;158:852-9.

20. Jolly K, Taylor R, Lip GY, Greenfield S, Raftery J, Mant J, et al. Home-based compared with hospital-based cardiac rehabilitation in a multi-ethnic population: costeffectiveness and patient adherence. The Birmingham Rehabilitation Uptake Maximisation Study (BRUM). Health Technol Assess 2007;11:1-118.

21. Terbizan DJ, Dolezal BA, Albano C. Validity of seven commercially available heart rate monitors. Meas Phys Educ Exerc Sci 2002;6:243-7. 